\title{
Dis-moi comment est ton aval et je te dirai comment marche ton accueil
}

\author{
Tell me how is your Hospital Admission Capacity and I will tell you How your ED Works
}

\author{
D. Pateron \\ (C) SFMU et Lavoisier SAS 2016
}

La surcharge des urgences est définie comme l'incapacité à certains moments d'assurer une prise en charge optimale de l'ensemble des patients présents dans un service des urgences (SU). L'explication de ce phénomène est trop souvent faussement résumée au nombre important de patients consultant aux urgences et qui pourraient être vus dans d'autres structures. De nombreux travaux ont bien montré que l'une des explications principales était en fait liée à la nature des patients pris en charge (complexité médicale ou médicosociale, âge élevé, nécessité d'une hospitalisation...) [1]. Ceci explique que les mois d'hiver, qui ne sont pas les plus chargés quantitativement, soient la période la plus compliquée pour les SU car les décompensations cardiorespiratoires des patients âgés augmentent le recours aux urgences de ce type de patients. La problématique tient avant tout à la difficulté de trouver un lit disponible en temps réel pour ces malades qui restent plusieurs heures, voire plusieurs jours au SU sur un brancard, ce que les Anglosaxons appellent les $E D$ boarders (EDB) [2].

Certains établissements tentent d'apporter des réponses concrètes à la nécessaire adaptation de la disponibilité de lits d'aval d'urgence en temps réel au besoin des SU : mise en place d'une gestion de l'hospitalisation, consultations posturgences, anticipation des sorties. En effet, le besoin d'hospitalisation à partir des urgences est prévisible. Elle nécessite l'engagement de la Commission Médicale d'Établissement et de la direction. Les services à vocation généraliste se sont réduits dans les hôpitaux, remplacés par des services spécialisés, créés au rythme de l'apparition de nouvelles spécialités ou sous-spécialités médicales ou chirurgicales. Parmi les services à vocation polyvalente, la médecine interne et la gériatrie n'échappent pas à l'expression légitime d'une volonté d'activité spécifique, avec ses effets négatifs vis-à-vis de l'offre d'hospitalisation en aval du SU.

\footnotetext{
D. Pateron $(\bowtie)$

Service des urgences, hôpital Saint-Antoine, Assistance Publique Hôpitaux de Paris, Université Pierre et Marie Curie, Paris,

184 rue du Faubourg Saint-Antoine, F-75012 Paris

e-mail : dominique.pateron@aphp.fr
}

Deux articles, dont un récemment parus dans les Annales Françaises de Médecine d'Urgence [3] et l'autre inclus dans ce numéro [4] rapportent les résultats d'une expérience instructive. Il s'agit d'unités d'aval des urgences dédiées comme réponse à cette problématique. Dans les deux cas, l'expérience semble avoir été positive pour l'amélioration des flux au sein des SU et sur la réduction du nombre d'EDB le matin. Si ces deux expériences enrichissent le débat, elles posent également certaines questions :

- ces unités sont-elles nécessaires ? Chaque établissement, siège d'un SU confronté à la surcharge (ne s'agit-il pas d'un pléonasme ?), doit faire l'analyse de ses difficultés : quelle fréquence, quelle durée, pour quel type de patients ; et doit trouver une adaptation de sa capacité d'aval pour les malades non programmés. Soit l'organisation historique de nos hôpitaux, où chaque service est « propriétaire » de ces lits et a un droit de regard sur les patients qui y sont admis, permet de répondre aux besoins de son SU, le plus souvent grâce à des conventions, et il n'y a aucune raison d'en changer, soit le SU est confronté régulièrement à la présence d'EDB et une organisation de la gestion des lits et un redimensionnement du parc de lits polyvalents de l'établissement s'imposent. Ceci peut être perçu comme une réponse par défaut et le révélateur d'un échec du système hospitalier classique, il n'en demeure pas moins une réflexion nécessaire et une piste utile ;

- ces unités doivent-elles répondre à des principes de fonctionnement communs ? Des règles ont été recensées par la Société Française de Médecine d'Urgence (SFMU) [5]. L'orientation en aval du SU ne doit se faire en unité polyvalente que si le diagnostic et/ou la prise en charge thérapeutique ne nécessitent pas le recours à des compétences et des techniques de spécialités. Dans l'étude réalisée par le CHU de Nantes, plus de la moitié des patients hospitalisés à partir du SU relevaient d'une prise en charge non spécialisée [4]. Le volume de ces unités doit être dimensionné pour répondre à la demande non spécialisée du SU et nécessite une analyse préalable des flux d'hospitalisation à partir du SU. Elles doivent comporter des lits d'hospitalisation ouverts en permanence, avec idéalement une 
possibilité d'ouverture transitoire de lits supplémentaires en cas d'afflux conjoncturel, notamment saisonnier (lits « gigognes » ou provisoires). Les référentiels de prise en charge des pathologies les plus fréquentes sont disponibles et actualisés de façon régulière, en collaboration avec les spécialistes de l'établissement, tenant compte de l'évolution des consensus et des recommandations professionnelles. Les patients admis dans l'unité proviennent en totalité du SU et ne sont pas programmés. L'admission d'un malade dans l'unité ne donne lieu à aucune négociation entre l'accueil et l'unité, dès lors que la décision d'hospitaliser a été prise par un senior du SU. Une durée de séjour aussi courte que possible est un objectif figurant explicitement dans le projet médical de l'unité. Pour y parvenir, il est nécessaire d'identifier, dès l'admission du patient, les procédures diagnostiques appropriées à la présente hospitalisation, et celles qui peuvent être différées et réalisables en externe. L'orientation précoce vers un service de soins de suite et de réadaptation (SSR) doit se faire dans le cadre d'un contrat. La séniorisation de la prise en charge est quotidienne. Aucun examen complémentaire n'est systématique. Des indicateurs d'évaluation sont mis en place dans le cadre d'une démarche qualité ;

- ces unités doivent-elles être administrativement ou contractuellement rattachées au SU et la continuité de soins doit-elle être assurée par le SU ? Il s'agit probablement de la question la plus difficile. Si les recommandations de la SFMU, fortement influencées par l'expérience de Nantes y sont favorables, les résultats des deux études présentent des résultats en opposition. L'équipe de Nantes tire avantage de cette unité pour une mutualisation et un renforcement du temps médical dédié au SU, tandis que pour l'équipe d'Antoine Béclère à Clamart, l'expérience a atteint certaines limites car du temps médical a dû être pris sur celui du SU pour assurer son fonctionnement. La relation fonctionnelle qui existe dans les deux cas entre le SU et l'unité d'aval des urgences pose également la question du métier de l'urgentiste. L'urgentiste, médecin des premières heures de prise en charge, voit son action s'étendre dans le temps au-delà des 24 heures pour résoudre un problème auquel l'institution n'a pas réussi à apporter de réponse. Quand proposera-t-on, pour des raisons fonctionnelles toutes aussi légitimes, l'unité de deuxième aval et le SSR de post-urgence rattachés au SU, et dans lesquelles les urgentistes seront impliqués ne serait-ce que pour assurer la continuité le week-end et pendant les vacances... À cet égard, mon expérience de membre du jury du concours de praticien hospitalier de médecine d'urgence m'a permis de constater que certains candidats se présentaient avec un projet exclusivement centré sur une activité d'aval, ce qui me semble poser problème. Si les praticiens affectés à ce type d'unité n'excluent pas a priori les urgentistes, ils doivent être issus de diverses filières (notamment médecine interne, médecine générale, mais aussi urgences, cardiologie, pneumologie, gériatrie...). Leur exercice doit être commun au sein de l'unité, dans le cadre de la prise en charge indifférenciée de tous les patients et les missions et les règles de fonctionnement me paraissent plus importantes que le rattachement au SU incluant la charge de la continuité de fonctionnement de ces unités.

En conclusion, la création ou la re-création d'unités de médecine polyvalente entièrement dédiées à l'aval des SU peut être très utile comme le montrent les expériences présentées $[3,4]$. Elles doivent correspondre à un besoin identifié et analysé au cas par cas dans chaque établissement. Les règles de fonctionnement de telles unités et de recrutement des praticiens y travaillant doivent être claires, partagées dans l'établissement. Le rattachement automatique au SU n'est pas nécessairement souhaitable pour ne pas éloigner les urgentistes de leur cœur de métier, même si l'intégration de ces unités à un pôle commun permet probablement de maintenir la pression sur la réalisation des missions de telles unités. Ce choix dépend plus de l'histoire de l'établissement et de son SU que de principes ou de dogmes.

\section{Références}

1. Guttmann A, Schull MJ, Vermeulen MJ, Stukel TA (2011) Association between waiting times and short term mortality and hospital admission after departure from emergency department: population based cohort study from Ontario, Canada. BMJ 342:d2983

2. American College of Emergency Physicians (2011) Definition of boarded patient. https://www.acep.org/Clinical-Practice-Management/Definition-of-Boarded-Patient-2147469010/ (Dernier accès le 15 avril 2016)

3. Andronikof M, Roche V, Civadier MS, et al (2016) Évaluation d'un service d'urgence possédant une unité d'hospitalisation conventionnelle. Ann Fr Med Urgence 6:85-92

4. Potel G, El Kouri D, Ngohou C, et al (2016) La médecine polyvalente : comment une médecine sobre peut-elle aider la médecine d'urgence? Ann Fr Med Urg 6:159-65

5. Société Française de Médecine d'urgence (SFMU) (2005) L'organisation de l'aval des urgences : état des lieux et propositions. http://www.sfmu.org/upload/referentielsSFMU/Aval_SU_SFMU_mai 2005.pdf (Dernier accès le 15 avril 2016) 